\title{
The Use of the Electronic Balance for Highly Accurate Direct Mass Measurements Without the Use of External Mass Standards
}

\section{R.M. Schoonover}

Frank E. Jones

U.S. DEPARTMENT OF COMMERCE Technology Administration

National Institute of Standards and Technology

Gaithersburg, MD 20899 



\section{The Use of the Electronic Balance for Highly Accurate Direct Mass \\ Measurements Without the Use of External Mass Standards}

R.M. Schoonover

Frank E. Jones

U.S. DEPARTMENT OF COMMERCE

Technology Administration

National Institute of Standards

and Technology

Gaithersburg, MD 20899

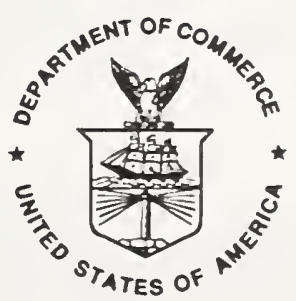

U.S. DEPARTMENT OF COMMERCE Ronald H. Brown, Secretary

TECHNOLOGY ADMINISTRATION

Mary L. Good, Under Secretary for Technology

NATIONAL INSTITUTE OF STANDARDS

AND TECHNOLOGY

Aratl Prabhakar, Director 

The Use of the Electronic Balance for Highly Accurate Direct

Mass Measurements

Without the Use of External Mass Standards

\author{
Randall Schoonover, NIST \\ Gaithersburg, MD \\ Frank E. Jones, NIST Retired \\ Potomac, Maryland
}

\title{
KEYWORDS
}

Calibration, Electronic balance, Mass, Traceability, Uncertainty, Weighing

\section{ABSTRACT}

The object of this paper is to show how to make highly accurate mass measurements using an appropriate electronic balance without the use of external mass standards. The method eliminates the need for a set of external mass standards and, therefore, the need to have these mass standards calibrated periodically at a standards laboratory. Traceability to NIST can be achieved by periodic calibration of the built-in mass standard of the balance by a standards laboratory and by self-verification of balance linearity and function. This method benefits the user with lower cost and a higher measurement rate. Presented here is a detailed analysis of the method, a determination of measurement uncertainty, and the traceability path. Lastly, the method is illustrated by specific examples including the calculation of uncertainty.

\section{INTRODUCTION}

The usual method of determining the mass of an $\mathrm{c}^{-}$ct is by comparing the nominally equal forces exerted on a balance pan by the object and by a mass standard (1). The small difference in mass between the unknown object and the mass standard is then expressed as the solution of two force equations which include terms for displacement volumes (i.e., densities) of the objects, and the air density. 
In this paper, we examine the concept ${ }^{(2,3)}$ that the mass of an object can be adequately determined (for most applications) by direct weighing on an electronic balance without the use of external standards. The only requirements are that the mass and density of the built-in weight of the balance be known adequately with respect to the SI units, and that the balance be linear or corrected for nonlinearity.

\section{THE FORCE DETECTOR}

The electronic balance can be considered to be a highly-linear and precise force detector. An overview of the electronic balance is given in $(2,4)$. A short summary of the principles of operation is given here. Detailed knowledge of the electronic circuits is unnecessary. Figure 1 illustrates the basic principles of a force balance, and a representative mechanical structure is shown in Figure 2.

When a downward force is applied to the balance pan (loaded with an object) it is opposed by a magnetic force generated by the interaction of two magnetic fields. One field is generated by a permanent magnet and the other by a controllable electromagnet. Usually, the magnetic force is applied through a multiplying lever and not by direct levitation. Sufficient magnetic force is generated to restore the mechanism (pan) to its unloaded position or null point relative to the balance structure. Obviously, the device is electromechanical and we should expect errors (both random and systematic) associated with both electrical and mechanical sources to arise in the use of these instruments. It is desirable in common weighing applications to tie the magnetic force to the unit of mass through calibration of the electronic circuit. The circuit is adjusted such that the algebraic sum of the gravitational and buoyant forces produces a balance indication approximately equal to the nominal value of the applied mass.

It is common practice for high-precision balances to be supplied with a built-in weight whose density is about $8 \mathrm{~g} / \mathrm{cm}^{3}$ and with its mass adjusted to a nominal value. This practice provides for a uniform response among balances to the given load at a given location. When the built-in calibration weight is tied to the mass unit by a traceable calibration, the electronic balance provides a convenient way to multiply and divide the mass unit within the capacity of the instrument. This built-in weight and the high degree of precision and linearity of the electronic force balance eliminates the need for a calibrated set of mass standards (i.e., a weight set).

In calibrating the balance, the manufacturer forces the no-load indication to be zero and, when the built-in calibration weight is engaged, adjusts its electronic circuit to indicate the nominal value of the built-in weight. The ideal balance response is, of course, a straight line connecting the no-load indication to the built-in weight indication. For some balances, linearity is preserved with extrapolation beyond these bounds. Usually, the response of balances is only approximately ideal and therefore, for some applications, observations not at calibrated points may require correction for 
nonlinearity. In the following discussion, it is assumed that the correction for nonlinearity has been applied to the balance observations for the unknown object. The subject of balance linearity will be discussed in detail later. However, we note that high-quality electronic balances are available for which linearity errors are less than 1 ppm of the capacity of the balance.

\section{DISCUSSION OF THE METHOD}

The calibration function of the analytical-quality electronic balance is totally or partially controlled by its microprocessor. In this calibration process, the pan-empty balance indication is set equal to zero. Likewise, the balance indication is adjusted to the nominal mass of the built-in weight when it is loaded on the balance. Customarily, the balance manufacturer adjusts the balance response to indicate the mass of the built-in weight (for the better balances) or the nominal mass of the built-in weight. This adjustment procedure ignores the opposing buoyant force on the built-in weight. In the latter case, the manufacturer adjusts the mass to be within one display count of the nominal mass.

During the calibration using the built-in weight, the balance response (indication) is proportional to the force imposed on the balance mechanism by the built-in weight. This force is expressed by the following equation:

$$
F_{S}=S\left(1-\frac{\rho_{a}}{\rho_{s}}\right) g=k O_{c}
$$

The force is $F_{s}, S$ is the mass of the built-in weight and $\rho_{s}$ is its density, $\rho_{a}$ is the density of air, $g$ is the local acceleration due to gravity, $k$ is a constant of proportionality, and $\mathrm{O}_{c}$ is the balance response in balance units.

Similarly, the force imposed on the balance pan by an object of unknown mass is expressed as:

$$
F_{x}=M_{x}\left(1-\frac{\rho_{a}}{\rho_{x}}\right) g=k\left(O_{L}-O_{E}\right)
$$

The force is $F_{x} ; M_{x}$ is the unknown mass of the object and $\rho_{x}$ is its density; $k$ is unchanged; $O_{L}$ is the balance response under load; and $O_{E}$ is the empty-pan balance indication. Usually, but not necessarily, $O_{E}$ is adjusted to zero at the beginning of the weighing process. It is imperative that the proportionality constant, $k$, remain unchanged for both the calibration cycle and the weighing cycle. The modern e tronic balance maintains its calibration, provided that the balance is left undisturbed and the environmental conditions are stable. 


$$
M_{x}=\frac{S\left[1-\frac{\rho_{a}}{\rho_{s}}\right]}{\left[\frac{O_{C}}{\left(O_{L}-O_{E}\right)}\right]\left[1-\frac{\rho_{s}}{\rho_{x}}\right]}
$$

We note that the quantity. $1 O_{C} /\left(O_{L}-O_{E}\right) l$ is the ratio of the force imposed on the balance by $S$ to the force imposed by $M_{x}$. Normally, $\rho_{s}$ and $\rho_{x}$ are known at some reference temperature, $t_{r}$. To obtain densities at the test temperature, $t$, corrections must be made for the expansion or contraction of the built-in weight and object using the following equation:

$$
\rho_{t}=\rho_{r} /\left[1+3 a\left(t-t_{r}\right)\right]
$$

where $a$ is the coefficient of linear thermal expansion of the material of which the built-in weight or object is constructed. We have used $\rho_{\mathrm{t}}$ to represent either $\rho_{\mathrm{s}}$ or $\rho_{\mathrm{x}}$ at the test temperature. Likewise, $\rho_{r}$ represents either density at the reference temperature. Either $\rho_{\mathrm{s}}$ or $\rho_{\mathrm{x}}$ can be determined using the balance by performing a hydrostatic weighings (3).

\section{LINEARITY TEST AND CORRECTION}

Ideally, the response of the balance would be linear; that is, observations would fall on the straight line between zero and the calibration point. For example, if an object of the same density as that of the calibration weight and $1 / 2$ its mass were placed on the pan of the balance, the balance would indicate $1 / 2$ the mass of the calibration weight. Failure to do this would indicate that the balance response is nonlinear. A linearity test should be performed to determine whether nonlinearity requires correction.

The linearity test involves dividing the mass range between zero and the calibration mass into four equal segments. This test requires four test weights, two at approximately $50 \%$ of the range and two at approximately $25 \%$ of the range. The test weights should be fabricated of the same material of which the built-in weight is 
fabricated in order to insure nearly equal densities. This effectively eliminates buoyancy terms in the following test.

We now assign mass values to the test weights relative to the mass of the built-in weight by sum and difference weighings. These weighings can be performed on the balance under test or on another balance. If the built-in weight can not be directly manipulated by the operator, it must be removed from the balance for this test.

We begin by comparing the sum of the two $50 \%$ weights with the built-in weight, after the balance has been calibrated. The comparison is performed by the method of substitution weighing ${ }^{(5)}$. Sum and difference weighings between the built-in weight, $S$, and the two $50 \%$ weights, designated $D$ and $E$, result in two equations:

$$
\begin{array}{r}
S-(D+E)=\Delta_{1} \\
D-E=\Delta_{2},
\end{array}
$$

where $\Delta_{1}$ and $\Delta_{2}$ are mass differences derived from balance indications. The solution of these two equations for $D$ is

$$
D=\left(S+\Delta_{2}-\Delta_{1}\right) / 2
$$

The quantity, $\Delta_{1}$, is the difference between the balance indication with $S$ on the balance pan and the balance indication with $D$ and $E$ on the balance pan. Similarly, $\Delta_{2}$, is the difference between the balance indication with $D$ on the pan and the balance indication with $E$ on the pan. The differences are small; therefore they are unaffected by reasonable balance nonlinearity. It is not necessary to solve for $E$.

This procedure is repeated for the two $25 \%$ weights, $F$ and $G$, where D or $E$ serves the function of $\mathrm{S}$ above. As above, the following two equations are solved for the $25 \%$ weight, F:

$$
\begin{gathered}
D-(F+G)=\Delta_{3} \\
F-G=\Delta_{4} .
\end{gathered}
$$

Solving these two equations for $F$ yields

$$
F=\left(D+\Delta_{4}-\Delta_{3}\right) / 2
$$

The uncertainties in the determinations of $D$ and $F$ relative to the built-in weight, $S$, can be reduced to trivial amounts by repeating the sequence of balance observations, $\mathrm{O}_{1}$ through $\mathrm{O}_{9}$. For example, if the sequence is repeated 15 additional times, the uncertainty in $D$ or $F$ is reduced by dividing each by the square root of 16 .

The linearity measurement sequence, where the weight on the pan is expressed as a percentage of the built-in weight, is: 
The $100 \%$ weight corresponds to both $50 \%$ weights being on the pan.

The observations, in mass units, corresponding to the above sequence are;

$$
\mathrm{O}_{1} \mathrm{O}_{2} \mathrm{O}_{3} \mathrm{O}_{4} \mathrm{O}_{5} \mathrm{O}_{6} \mathrm{O}_{7} \mathrm{O}_{8} \mathrm{O}_{9}
$$

This measurement sequence minimizes the effects of drift and hysteresis, if any.

The linearity correction is derived relative to the sum of the $50 \%$ weights, that is, at the $100 \%$ calibration point. The linearity correction at the $50 \%$ point, $\mathrm{LC}_{50 \%}$, is

$$
L C_{50 \%}=D-\left[\left(O_{5}-O_{3}\right)+\left(O_{5}-O_{7}\right)\right] / 2
$$

The linearity correction at the $25 \%$ point is

$$
L C_{25 \%}=F-\left[\left(O_{5}-O_{4}\right)+\left(O_{5}-O_{6}\right)\right] / 2
$$

The linearity correction at the $75 \%$ point is

$$
L C_{75 \%}=(D+F)-\left[\left(O_{5}-O_{2}\right)+\left(O_{5}-O_{8}\right)\right] / 2
$$

With the linearity correction determined at 5 points (it is zero at the $0 \%$ and $100 \%$ points), the shape of the linearity correction-mass curve is revealed by plotting the 5 points against mass. Intermediate points can be determined graphically or by mathematically fitting a curve to the points. In all cases, the linearity corrections (if significant) are subsequently added to or subtracted from the mass calculated from the balance indication. For those balances that extrapolate beyond the calibration point the same technique can be applied to the extrapolated region. One only needs to load the balance pan with a weight of known mass nominally equal to $S$ and then perform the linearity test as described above. There are hybrid balances that use a series of tare weights (built-in weights) in conjunction with the electronic force balance to increase the capacity. These weights require calibration by the method described in (6) to assure linearity.

\section{UNCERTAINTIES}

The measurement uncertainties are propagated by the method described by $\mathrm{Ku}^{(7)}$. The general propagation equation for the uncertainty of $M_{x}$ is: 


$$
\left(S D_{M_{x}}\right)=\left[\sum_{i=1}^{n}\left[\frac{\partial M_{x}}{\partial y_{i}}\right]^{2}\left(S D_{i}\right)^{2}\right]^{\frac{1}{2}}
$$

where $\partial M_{x} / \partial y_{i}$ is the partial derivative of the equation for the unknown mass, $M_{x}$, with respect to the parameter, $Y_{i} \cdot S D_{i}$ is the estimate of standard deviation for each parameter, $y_{i}$. SD's in this paper are $u_{a}, u_{b}$ or $u_{c}$ as defined in NIST Technical Note $1297^{(8)}$. The parameters are $\mathrm{S}, \rho_{\mathrm{s}}, \rho_{\mathrm{x}}, \rho_{\mathrm{a}}, \mathrm{O}_{\mathrm{C}}, \mathrm{O}_{\mathrm{L}}$, and $\mathrm{O}_{\mathrm{E}}$. The parameter uncertainties for $S, \rho_{5}$ and $\rho_{x}$ are Type B uncertainties; the remaining are Type A. Equation ( 8 ) is consistent with NIST TECHNICAL NOTE 1297. Second-order effects such as the thermal expansion or contraction of the weights, and the covariance terms in the $\mathrm{Ku}$ equation are considered negligible. Referring now to equation (3), the partial derivatives are:

$$
\begin{aligned}
& \frac{\partial M_{x}}{\partial S}=\frac{\left[1-\frac{\rho_{a}}{\rho_{s}}\right]}{\left[\frac{O_{C}}{O_{L}-O_{E}}\right]\left[1-\frac{\rho_{a}}{\rho_{x}}\right]} \\
& \frac{\partial M_{x}}{\partial O_{C}}=-S\left[\frac{\left(1-\frac{\rho_{a}}{\rho_{S}}\right)}{\left(1-\frac{\rho_{a}}{\rho_{x}}\right)}\right]\left[\frac{\left(O_{L}-O_{E}\right)}{O_{C}^{2}}\right] \\
& \frac{\partial M_{x}}{\partial\left(O_{L}-O_{E}\right)}=\frac{S\left[1-\frac{\rho_{s}}{\rho_{s}}\right]}{O_{C}\left[1-\frac{\rho_{a}}{\rho_{x}}\right]} \\
& \frac{\partial M_{x}}{\partial \rho_{s}}=\frac{\left[\frac{S \rho_{a}}{\rho_{s}^{2}}\right]}{\left[\frac{O_{C}}{O_{L}-O_{E}}\right]\left[1-\frac{\rho_{e}}{\rho_{x}}\right]}
\end{aligned}
$$




$$
\frac{\partial M_{x}}{\partial \rho_{s}}=-\left[\left(\frac{1}{\rho_{s}}\right)\left(1-\frac{\rho_{s}}{\rho_{x}}\right)+\left(\frac{1}{\rho_{x}}\right)\left(1-\frac{\rho_{s}}{\rho_{s}}\right)\right]\left[\frac{S}{\left[\frac{O_{C}}{O_{L}-O_{E}}\right]\left[1-\frac{\rho_{s}}{\rho_{x}}\right]^{2}}\right]
$$

$$
\frac{\partial M_{x}}{\partial \rho_{x}}=\left[\frac{\left(\frac{\rho_{s}}{\rho_{x}^{2}}\right)}{\left(1-\frac{\rho_{s}}{\rho_{x}}\right)}\right]\left[\frac{s\left[1-\frac{\rho_{s}}{\rho_{s}}\right]}{\left[\frac{O_{c}}{\left(O_{L}-O_{E}\right)}\right]}\right]
$$

\begin{tabular}{|c|c|c|c|c|}
\hline$y_{i}$ & Value & SD & $\partial M_{x} / \partial y_{i}$ & {$\left[S D\left(y_{i}\right)^{2}\left[\frac{\partial M_{x}}{\partial y_{i}}\right]^{2}\right]^{1 / 2}$} \\
\hline $\mathrm{s}$ & $100 \mathrm{~g}$ & $0.000050 \mathrm{~g}$ & 2.00073 & $0.00010 \mathrm{~g}$ \\
\hline$\rho_{\mathrm{s}}$ & $8 \mathrm{~g} / \mathrm{cm}^{3}$ & $\begin{array}{c}0.00032 \\
\mathrm{~g} / \mathrm{cm}^{3}\end{array}$ & $\begin{array}{c}0.00375 \\
\mathrm{~cm}^{3}\end{array}$ & $0.0000012 \mathrm{~g}$ \\
\hline$\rho_{x}$ & $2.329 \mathrm{~g} / \mathrm{cm}^{3}$ & $\begin{array}{c}0.000004 \\
\mathrm{~g} / \mathrm{cm}^{3}\end{array}$ & $\begin{array}{c}0.04426 \\
\mathrm{~cm}^{3}\end{array}$ & $0.00000018 \mathrm{~g}$ \\
\hline $\mathrm{O}_{c}$ & $100 \mathrm{~g}$ & $0.000049 \mathrm{~g}$ & 2.00073 & $0.000056 \mathrm{~g}$ \\
\hline$O_{L}-O_{E}$ & $200 \mathrm{~g}$ & $0.000138 / N 6$ & 1.000366 & $0.000056 \mathrm{~g}$ \\
\hline \multirow[t]{2}{*}{$\rho}$. & $\begin{array}{l}0.0012 \\
\mathrm{~g} / \mathrm{cm}^{3}\end{array}$ & $\begin{array}{c}0.000000086 \\
\mathrm{~g} / \mathrm{cm}^{3}\end{array}$ & $\begin{array}{c}-60.87659 \\
\mathrm{~cm}^{-3}\end{array}$ & $0.000052 \mathrm{~g}$ \\
\hline & & & RSS $=$ & $\begin{array}{r}0.00016 \mathrm{~g} \\
(0.8 \mathrm{ppm}) \\
\end{array}$ \\
\hline
\end{tabular}

\section{TABLE 1}

For a typical weighing, the weighing of a 200-g silicon crystal, Table 1 lists the values for the estimates of standard deviation (SD), the partial derivatives, and their products. The root-sum-square (RSS) of the products is the estimate of the uncertainty in the determination of $M_{x}$ with a coverage factor of 1 . The SD of $S$ is the 
calibration uncertainty (provided by a standards laboratory, for example) with a coverage factor of 1 . The SD's of $\rho_{\mathrm{s}}$ and $\rho_{\mathrm{x}}$ are similarly provided or determined, again with a coverage factor of 1 . The value of $\left(\mathrm{O}_{L}-\mathrm{O}_{E}\right)$ in the table is the mean of repeated determinations performed at one sitting. The SD of the mean value is the $S D$ determined from the repeated determinations divided by the square root of the number, $n$, of repeated determinations, 6 in this case. The SD of $O_{c}$ is the SD of the balance with a 100-g load; this cannot be reduced by repeating the automatic balance calibration process. The SD of a single determination of $\left(O_{L}-O_{E}\right)$ is the same as the $S D$ of $O_{c}$, that is, the same as the SD of the balance.

In the example above, the SD of the lower half of the balance range is constant. In the better balances, this is in general not true; the SD is smaller at the lower end of the range of the balance and the user might wish to take advantage of this fact. We determined the balance SD and linearity correction for the $200 \mathrm{~g}$ load from a set of 6 weighings. The mean SD of the balance was combined with the mean SD of the linearity correction by the RSS method for an estimate of the effective SD. The linearity correction measurements were also performed 6 times.

\section{BALANCE SELECTION}

The parameters in Table 1 which contribute the dominant uncertainties for a mass determination within the capacity of the balance are the SD of the balance $\left(O_{c}\right)$ and $S$. The uncertainty of $S$ can be reduced significantly by a rigorous calibration. Depending on the desired accuracy for mass determinations, a balance with a lesser or greater SD might be chosen. After the SD on $S$ and $\mathrm{O}_{c}$, the limiting parameter becomes $\rho_{\mathrm{a}}$. In selecting a balance, the error propagation table, Table 1 , is useful in determining the desired SD of a balance. Having determined the desired SD, one then depends on the specified SD's provided by manufacturers to select an appropriate balance.

\section{Determining the Estimate of Standard Deviation, SD, of the Balance}

Having acquired an appropriate balance, one then should determine, and use, the SD of the balance in calculating weighing uncertainties. The SD can be determined by multiple weighings of the object to be weighed, or of any stable weight within the range of the balance capacity. It is preferred that the mass of the stable weight be near that of the object(s) to weighed. The SD is determined in the usual manner from the values from the multiple weighings. Returning now to Table 1, the SD to be assigned to $O_{C}$ and to $\left(O_{L}-O_{E}\right)$ is that determined above. The SD assigned to $O_{C}$ cannot be reduced by $n$ repeated calibrations, that is, is cannot be divided by the square root of $n$. However, the SD assigned to $\left(O_{L}-O_{E}\right)$ can be so reduced.

\section{DATA}


Five independent determinations of the mass of a 200-g silicon crystal were made using an electronic force balance and the method described here. The five determinations of the mass of the crystal, in grams, are listed below:

199.4266, 199.4264, 199.4267, 199.4266, 199.4273

The mean of these values is $199.42672 \mathrm{~g}$, and the estimate of standard deviation $(S D)$ is $0.00034 \mathrm{~g}$. The relative standard deviation is 1.7 parts per million. The SD is about two times as large as our predicted uncertainty (Table 1). The lack of thermal equilibrium of the object and the surrounding air is the major cause of this small difference.

The quantitative success of the above measurements can be misleading in the conventional use of the electronic balance since in the example the densities of the objects are very well known. If the densities of $S$ and $M_{x}$ were only known to 1 part per thousand (a crude density determination), a table similar to Table 1 would yield a predicted uncertainty of 1 part per million rather than 0.8 part per million.

\section{DISCUSSION}

We have shown that very accurate measurements of mass can be achieved by the proper use of an appropriate electronic balance. In this treatment, the need for calibrated laboratory weight sets is eliminated. Traceability to NIST is attained through calibration of the built-in calibration weight of the electronic balance by NIST or by other standards laboratories. Both the mass and density of the built-in weight should be provided by the standards laboratory. In most cases, a balance manufacturer can supply limits on the nonlinearity and the estimate of standard deviation (SD) of the balance, and the density and the mass and uncertainty of the calibration built-in weight. If one accepts the values of the nonlinearity and SD provided by the manufacturer, the uncertainties in these two quantities must be combined by root sum square to calculate the estimated effective balance SD.

We have shown how to make determinations of nonlinearity and of the SD of the balance. In the latter case, either an object to be weighed or another object of stable mass is used; a standard of mass is not required. We have shown in detail how to estimate and propagate uncertainties. The calculation and propagation of uncertainties have been demonstrated using data from weighings of an object of density $2.329 \mathrm{~g} / \mathrm{cm}^{3}$.

\section{Direction of Future Developments in Electronic Balances and Uses}

One of the present shortcomings of electronics balances is the fact that repeated calibrations of the balance using the built-in weight cannot be averaged. As presently configured, $\mathrm{O}_{c}$ is the result of only one calibration. Therefore, the uncertainty in $\mathrm{O}_{c}$ cannot be reduced by the square root of the number of repeated calibrations. 
Balance manufacturers have the opportunity of making a great step forward by incorporating a hollow (or low-density) weight ${ }^{(9)}$ in the balance so that air density might be determined automatically by weighings of the built-in weight and the hollow (or low-density) weight. This would eliminate the inconvenient and expensive practice of measuring pressure, temperature, and relative humidity to calculate air density. The algorithm for determining air density using the two weights and incorporating the air density to make an automatic buoyancy correction could be incorporated in the microprocessor of the balance. The balance would then query the user for the density of the object, the mass of which is being determined. And thus, the balance would indicate mass directly, rather than an approximate mass uncorrected for air buoyancy in case the densities of the weighed object and built-in weight are not equal.

\section{REFERENCES}

(1) Schoonover, Randall M., and Jones, Frank E., "Examination of Parameters that Can Cause Errors in Mass Determinations," NISTIR 5376, National Institute of Standards and Technology, February, 1994.

(2) Schoonover, Randall M., et al., "The Electronic Balance and Some Gravimetric Applications," ISA 1993 Proceedings of the $39^{\text {th }}$ International Instrumentation Symposium, (1993), 299-320.

(3) Schoonover, Randall M., et al., "The Determination of Density of Mass Standards; Requirement and Method," Measurement Science Conference, Pasadena, CA, January, 1994.

(4) Schoonover, Randall M., "A Look at the Electronic Analytical Balance," Anal. Chem. (1992) 973A-980A.

(5) Jaeger, Klaus B., and Davis, Richard S., "A Primer for Mass Metrology," NBS Special Publication 700-1, Industrial Measurement Series, (1984).

(6) Schoonover, Randall M., and Jones, Frank E., "Air Buoyancy Correction In HighAccuracy Weighing On Analytical Balances," Anal. Chem. (1981) 900-902.

(7) Ku, Harry S.; "Statistical Concepts in Metrology," Handbook of Industrial Metrology, Chapter 3, American Society of Tool and Manufacturing Engineers, Prentice-Hall, NY, (1967).

(8) Taylor, B.N., Kuyatt, C.E., "Guidelines for Evaluating and Expressing the Uncertainty of NIST Measurement Results," NIST Technical Note 1297.

(9) Schoonover, Randall M., "A Simple Gravimetric Method to Determine Barometric Corrections", J. Nat. Bur. Stand. (U.S.), 85, No. 5, (1980), 341-345. 
(10) Davis, Richard S., "Equation for the Determination of the Density of Moist Air (1981/91)," Metrologia (1992), 29.

(11) Jones, Frank E., "The Air Density Equation and the Transfer of the Mass Unit," J. Res. Nat. Bur. Stand. (U.S.) 83, No. 5, (1978), 419-428. 


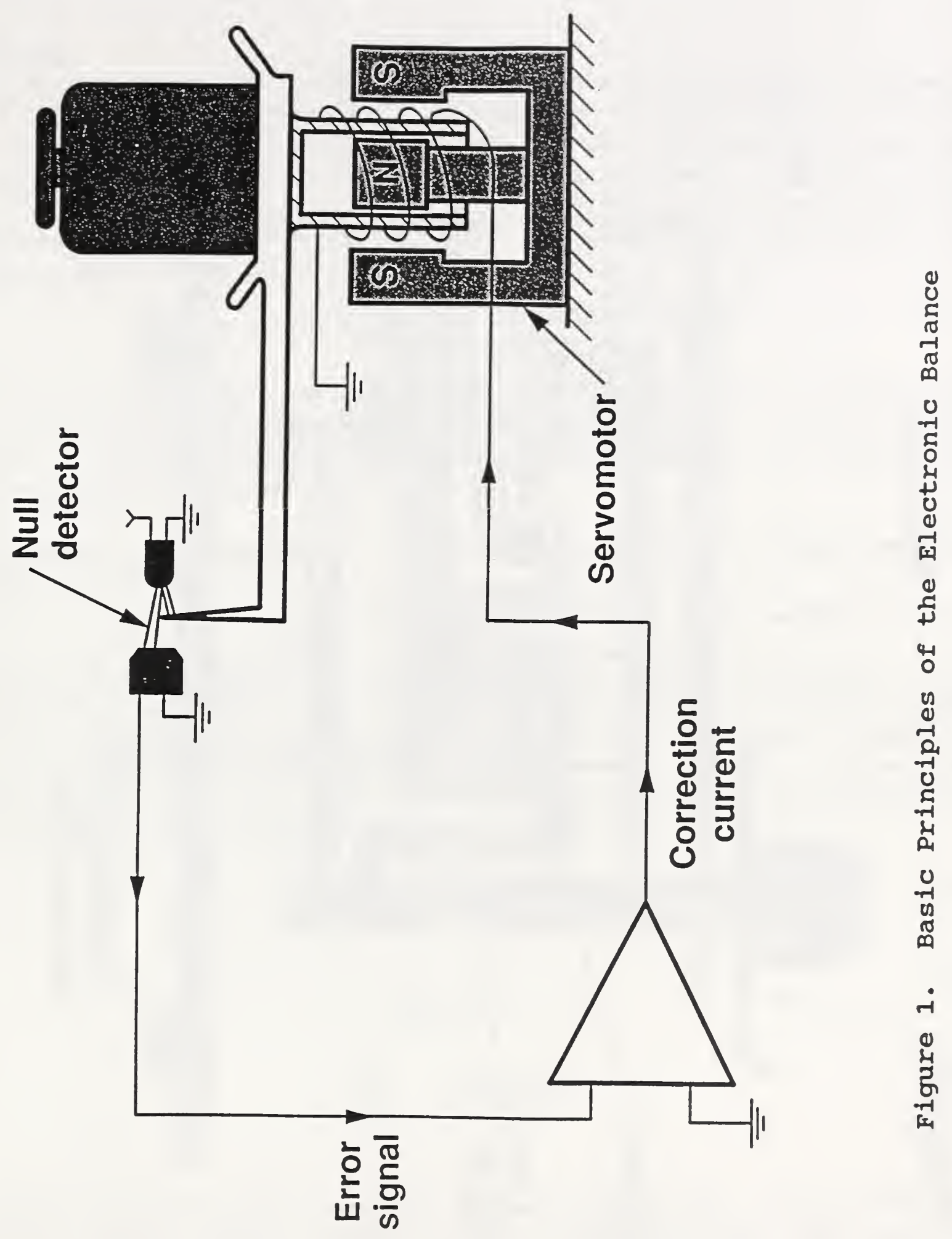




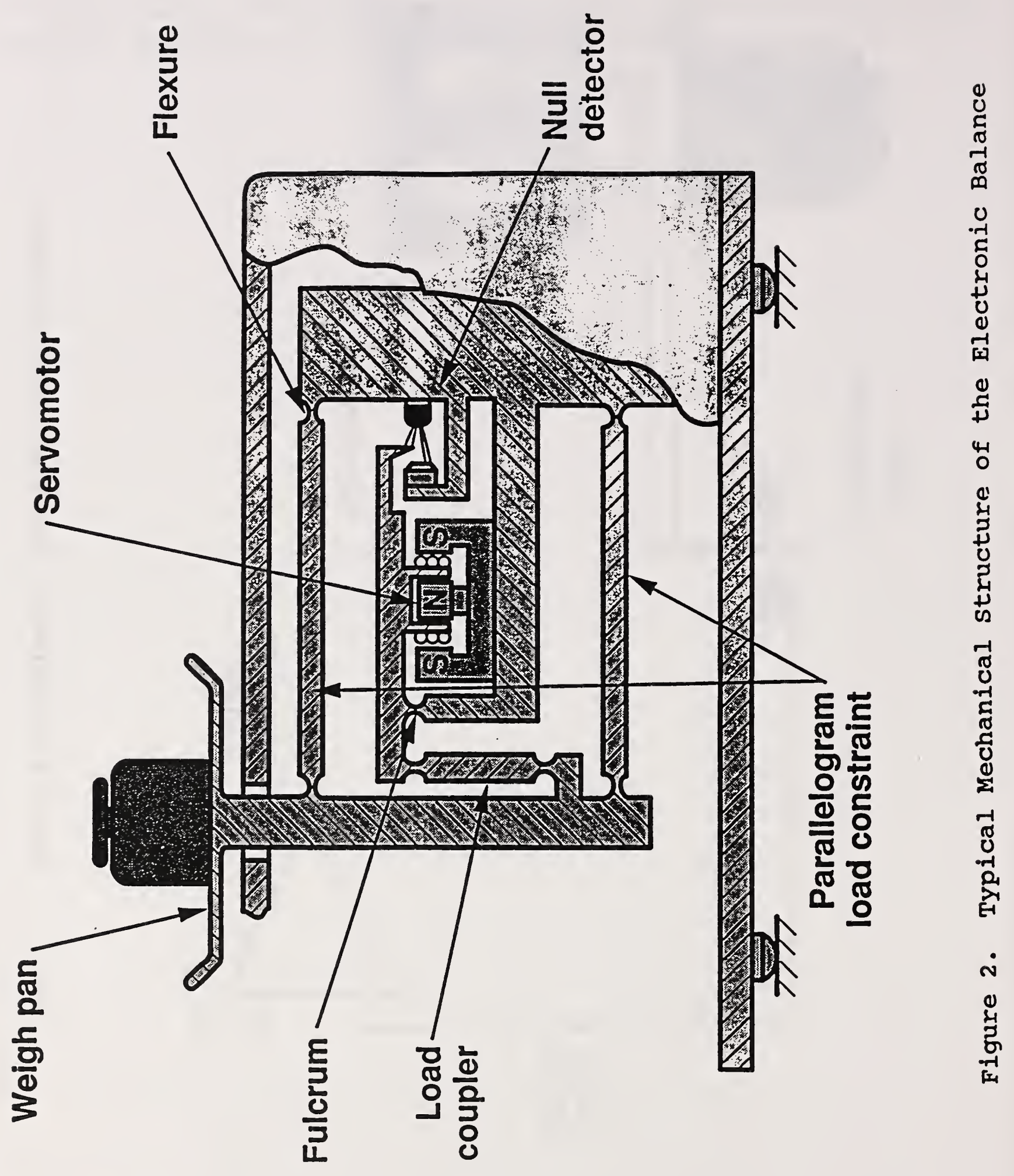




\section{APPENDIX}

\section{Air Density}

The air density equation for moist air used in state-of-the-art mass determinations is the CIPM 1981/91 recommendation ${ }^{(10)}$. This equation ties its predecessor, CIPM-81, to the International Temperature Scale of 1990 (ITS-90) and utilizes better estimates for the values of some of the constants and other parameters. The equation is tedious to use and its use is unnecessary for accurate mass measurements where the desired uncertainty is less stringent than 0.001 part per million. A much easier to use formula, based on the work of Jones ${ }^{(9,11)}$, is given here:

$$
\rho_{s}=\left(\frac{0.0034836}{T Z}\right)\left(P-0.0037960 \cup e_{s}\right)
$$

where $\rho_{\mathrm{a}}$ is in $\mathrm{mg} / \mathrm{cm}^{3}$ and is converted to $\mathrm{g} / \mathrm{cm}^{3}$ by dividing by 1000 . The parameters are the barometric pressure, $P$, in pascals, air temperature, $T$, in kelvins, relative humidity, $U$, in percent, the saturation vapor pressure of moist air, $e_{s}$, in pascals, and the compressibility factor for moist air, $\mathbf{Z}$.

The parameter $e_{s}$ is dependent on air temperature and can be calculated from the formula given here

$$
e_{s}=1.7526 \times 10^{11} e^{(-5315.56 / T)}
$$

or determined from Table 1A. The compressibility factor $\mathbf{Z}$ depends on the air temperature, pressure and relative humidity and can be easily determined from Table 2A. The user can estimate the uncertainty associated with the use of the above air density equation from estimates of uncertainty in the measurements of temperature, pressure and relative humidity by evaluating partial derivatives. The partial derivatives of the air density with respect to the above parameters are:

$$
\begin{gathered}
\frac{\partial \rho_{e}}{\partial T}=-\frac{\rho_{a}}{T} \\
\frac{\partial \rho_{a}}{\partial P}=\frac{\rho_{a}}{P} \\
\frac{\partial \rho_{a}}{\partial U}=-\frac{0.034970}{T}
\end{gathered}
$$

In deriving the air density equation the mole fraction of $\mathrm{CO}_{2}$ in the air has been assigned a constant value of 0.00043 . A set of partial derivatives that include the partial with respect to $\mathrm{CO}_{2}$ is given in (9). An example of error propagation is given in Table 3A. The parameter SD's are our uncertainty estimates for measurements of $T, P$ and $U$ in the balance weighing chamber during the weighing of the $200-\mathrm{g}$ silicon crystal. The RSS value is used in Table 1 of the text as the SD for the parameter $\rho_{\mathrm{a}}$. 
In Table 3A, we give our estimates of the uncertainties that can be achieved for these parameters and have evaluated the partial derivatives. The covariances are assumed to be negligible and are therefore not given. The root sum square (RSS) uncertainty is carried forward as the air density uncertainty (type A). The type B uncertainty arising from the constant parameters in the air density equation is insignificant. 


\begin{tabular}{||c|c|c|c|c|c|c||}
\hline \hline & 20 & 21 & 22 & 23 & 24 & 25 \\
\hline .00 & 2390 & 2487 & 2644 & 2810 & 2985 & 3169 \\
\hline .05 & 2346 & 2495 & 2652 & 2818 & 2994 & 3178 \\
\hline .10 & 2353 & 2503 & 2669 & 2827 & 3003 & 3188 \\
\hline .15 & 2360 & 2510 & 2669 & 2836 & 3012 & 3197 \\
\hline .20 & 2367 & 2510 & 2677 & 2844 & 3021 & 3207 \\
\hline .25 & 2375 & 2526 & 2685 & 2853 & 3030 & 3216 \\
\hline .30 & 2382 & 2533 & 2693 & 2861 & 3030 & 3226 \\
\hline .35 & 2390 & 2541 & 2701 & 2870 & 3048 & 3235 \\
\hline .40 & 2397 & 2549 & 2709 & 2870 & 3057 & 3245 \\
\hline .45 & 2404 & 2557 & 2718 & 2887 & 3066 & 3255 \\
\hline .50 & 2412 & 2565 & 2776 & 2836 & 3075 & 3264 \\
\hline .55 & 2419 & 2573 & 2734 & 2905 & 3085 & 3274 \\
\hline .60 & 2427 & 2580 & 2743 & 2914 & 3094 & 3284 \\
\hline .65 & 2434 & 2588 & 2751 & 2827 & 3103 & 3294 \\
\hline .70 & 2442 & 2596 & 2759 & 2931 & 3112 & 3363 \\
\hline .75 & 2449 & 2604 & 2768 & 2940 & 3122 & 3313 \\
\hline .80 & 2457 & 2612 & 2776 & 2949 & 3131 & 3323 \\
\hline .85 & 2464 & 2620 & 2785 & 2958 & 3140 & 3333 \\
\hline .50 & 2472 & 2628 & 2793 & 2967 & 3150 & 3343 \\
\hline .95 & 2480 & 2636 & 2801 & 2976 & 3159 & 3353 \\
\hline \hline
\end{tabular}

Table 1A 


\begin{tabular}{|c|c|c|c|c|c||}
\hline \multicolumn{6}{|c|}{ COMPRESSIBILITY FACTOR, Z } \\
\hline Temperature & \multicolumn{2}{|c|}{ Pressure } & \multicolumn{3}{c|}{ Relative Humidity } \\
\hline Celsius & pascals & $\mathrm{mm}$ & $25 \%$ & $50 \%$ & $75 \%$ \\
\hline 20 & 80000 & 600 & 0.99963 & 0.99967 & 0.99965 \\
\hline- & 100000 & 750 & 0.99962 & 0.99960 & 0.99959 \\
\hline 21 & 80000 & 600 & 0.99970 & 0.99968 & 0.99966 \\
\hline- & 100000 & 750 & 0.99963 & 0.99961 & 0.99959 \\
\hline 22 & 80000 & 600 & 0.99971 & 0.99969 & 0.99966 \\
\hline- & 100000 & 750 & 0.99964 & 0.99962 & 0.99960 \\
\hline 23 & 80000 & 600 & 0.99971 & 0.99969 & 0.99966 \\
\hline- & 100000 & 750 & 0.99964 & 0.99962 & 0.99959 \\
\hline 24 & 80000 & 600 & 0.99972 & 0.99970 & 0.99967 \\
\hline- & 100000 & 750 & 0.99965 & 0.99969 & 0.99961 \\
\hline 25 & 80000 & 600 & 0.99972 & 0.99970 & 0.99967 \\
\hline- & 100000 & 750 & 0.99966 & 0.99964 & 0.99961 \\
\hline
\end{tabular}

Table 2A.

\begin{tabular}{|l|l|l|l|l||}
\hline Variable & Value & $\mathrm{SD}\left(\mathrm{y}_{\mathrm{i}}\right)$ & $\begin{array}{l}\text { Partial } \\
\text { Derivative }\end{array}$ & $\begin{array}{l}\mathrm{SD}\left(\rho_{\mathrm{a}}\right)_{i \prime} \\
\mathrm{g} / \mathrm{cm}^{3}\end{array}$ \\
\hline $\begin{array}{l}\text { Temp. } \\
\text { (T) }\end{array}$ & $\begin{array}{l}296 \mathrm{~K} \\
\left(23^{\circ} \mathrm{C}\right)\end{array}$ & $\begin{array}{l}0.02 \mathrm{~K} \\
\left(0.0{ }^{\circ} \mathrm{C}\right)\end{array}$ & 0.0000041 & $\begin{array}{l}0.082 \\
\times 10^{-6}\end{array}$ \\
\hline $\begin{array}{l}\text { Press. } \\
\text { (P) }\end{array}$ & $\begin{array}{l}100258 \mathrm{~Pa} \\
(752 \mathrm{~mm} \\
\mathrm{Hg})\end{array}$ & $\begin{array}{l}65 \mathrm{~Pa} \\
(0.5 \mathrm{~mm} \\
\mathrm{Hg})\end{array}$ & 0.000000012 & $\begin{array}{l}0.78 \times \\
10^{-8}\end{array}$ \\
\hline $\begin{array}{l}\text { Rel. } \\
\text { Hum. } \\
\text { (U) }\end{array}$ & $41 \%$ & $3 \%$ & -0.00000012 & $\begin{array}{l}0.36 \times \\
10^{-6}\end{array}$ \\
\hline & & & & \multicolumn{2}{|c|}{$\mathrm{RSS}=$} \\
\hline
\end{tabular}

Table 3A. 
